MS-TPI-98-13

\title{
Monte Carlo Algorithms For the Fully Frustrated XY Model
}

\author{
S. Große Pawig and K. Pinn \\ Institut für Theoretische Physik I, Universität Münster \\ Wilhelm-Klemm-Str. 9, D-48149 Münster, Germany \\ e-mail:pawig@math.uni-muenster.de,pinn@uni-muenster.de
}

\begin{abstract}
We investigate local update algorithms for the fully frustrated XY model on a square lattice. In addition to the standard updating procedures like the Metropolis or heat bath algorithm we include overrelaxation sweeps, implemented through single spin updates that preserve the energy of the configuration. The dynamical critical exponent (of order two) stays more or less unchanged. However, the integrated autocorrelation times of the algorithm can be significantly reduced.
\end{abstract}




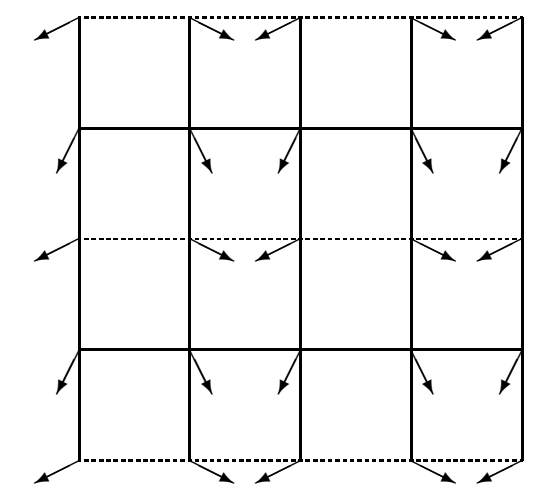

Figure 1: A ground state configuration of the FFXY model. The dotted lines represent links with antiferromagnetic couplings, the full lines are links with ferromagnetic couplings.

\section{Introduction}

The 2-dimensional fully frustrated XY (FFXY) model has attracted a lot of attention over the years. It is defined through the partition function

$$
Z=\int \prod_{i}\left(d^{2} s_{i} \delta\left(\vec{s}_{i}^{2}-1\right)\right) \exp \left[\beta \sum_{<i j>} J_{i j} \vec{s}_{i} \cdot \vec{s}_{j}\right] .
$$

The 2-component spins $s_{i}$ are defined on a square grid. They are constrained to unit length, ${\overrightarrow{s_{i}}}^{2}=1$. $\beta>0$ denotes the inverse temperature. Units are chosen such that $k_{B} \equiv 1$. The $J_{i j}$ are either +1 or -1 in such a way that each elementary plaquette of the lattice contains exactly one antiferromagnetic bond (with $J=-1$ ). We stick to the convention that the antiferromagnetic links are those lying in every second row of the lattice, see figure 1, which shows also one of the ground states of the FFXY model.

The importance of the frustrated XY model stems from the fact that it provides a convenient framework to study a variety of interesting phenomena displayed by numerous physical systems. Experimental realizations of the model are, e.g., 2-dimensional arrays of Josephson junctions or superconducting wire networks [1, 2, 3].

The phase structure of the FFXY model is still debated. In the literature, there seems to be agreement about the following: In addition to the type of 
excitations present in the unfrustrated XY model [⿴] (spin waves at large $\beta$ and vortices for $\beta \leq \beta_{K T}$ ) there is a new type of discrete excitations which should be relevant at sufficiently large $\beta$. With each elementary plaquette $i$ one can associate a chirality variable $\sigma_{i}$, defined through

$$
\begin{aligned}
\sigma_{i} & =\operatorname{sign}\left(\sin \phi_{12}+\sin \phi_{23}+\sin \phi_{34}+\sin \phi_{41}\right), \\
\phi_{k l} & =\theta_{k}-\theta_{l}-A_{k l} .
\end{aligned}
$$

The corners of the plaquette $i$ are labelled clockwise $1 \ldots 4$. The angles $\theta_{k}$ are defined through the relation $\vec{s}_{k}=\left(\cos \theta_{k}, \sin \theta_{k}\right)$, and $A_{k l}=0$ or $\pi$ for ferromagnetic and antiferromagnetic links, respectively. $\sigma_{i}$ can assume values \pm 1 . The global symmetry of the model with respect to the reflection of all $\sigma$ 's can be spontaneously broken. If this transition takes place at a $\beta$ value different from that where the XY degrees of freedom undergo a (most likely) Kosterlitz-Thouless phase transition, then it follows from standard wisdom about universality that it should belong to the universality class of the 2-dimensional Ising model. On the other hand, if the two transitions are exactly on top of each other, one expects a new universality class, with exponents different from the $2 \mathrm{D}$ Ising values.

The critical properties of the FFXY model have been investigated in a number of Monte Carlo studies, see, e.g., refs. [5]-16]. All studies, including the most recent ones, indicate that there is an urgent need to go to larger lattices. Unfortunately, the simulations of the FFXY model exhibit strong critical slowing down. On large lattices one has to perform many update sweeps in order to obtain a statistically independent configuration. E.g., at $\beta=2.2$, which is in the critical region, the autocorrelation time of a local Metropolis algorithm is $\approx 1600$ on a 64 by 64 lattice. Fortunately, there seems to be no exponential slowing down. At criticality the autocorrelations grow like $\tau \simeq A L^{z}$, where $L$ denotes the linear lattice extension. The dynamical critical exponent is about 2.3 for a local Metropolis update algorithm.

It is tempting to apply cluster algorithms [17, 18] to the problem. However, as has been experienced quite often in the case of frustrated models, there is no obvious way to avoid cluster size distributions with strong weight on very large cluster sizes, thus spoiling any gain in efficiency. Using the general formalism of Kandel et al. [19], with the useful extensions of ref. [20], one can convince oneself that (distinct from the case of the fully frustrated Ising model [21]) in a quite large class of algorithms there is none that avoids this problem. 
In ref. [8] an attempt was made to improve on the performance of the usual local heat bath or Metropolis algorithm, employing Fourier acceleration. However, there was negligible speedup of observables built from the discrete $\sigma$-variables introduced above. In ref. [9] a Hybrid Monte Carlo algorithm was used, but without a quantification of critical slowing down.

We here investigate the inclusion of microcanonical overrelaxation steps [22]-29] in the update procedure. To the best of our knowledge it has not yet been employed in the context of the frustrated XY model. The algorithm is very easy to implement, keeps the updating perfectly local and thus allows simple vectorization and/or parallelization. Still, an effective speedup of one order of magnitude can be obtained.

\section{Observables and Algorithms}

In order to test a number of algorithms, we measured for both the XY degrees of freedom $\vec{s}$ and the Ising type variables $\sigma$ the energies,

$$
\begin{aligned}
E_{\mathrm{XY}} & =\left\langle\overrightarrow{s_{i}} \cdot \overrightarrow{s_{j}}\right\rangle, \\
E_{\mathrm{I}} & =\left\langle\mu_{i} \mu_{j}\right\rangle .
\end{aligned}
$$

Here, $i$ and $j$ are nearest neighbours in the lattice, and the variables $\mu_{i}$ are defined through

$$
\mu_{i}=(-1)^{i_{x}+i_{y}} \sigma_{i},
$$

where the lattice coordinate $i$ has components $\left(i_{x}, i_{y}\right)$. Furthermore, we measured the susceptibilities

$$
\begin{aligned}
\chi_{\mathrm{XY}} & =\sum_{j}\left\langle\overrightarrow{s_{i}} \cdot \overrightarrow{s_{j}}\right\rangle, \\
\chi_{\mathrm{I}} & =\sum_{j}\left\langle\mu_{i} \mu_{j}\right\rangle .
\end{aligned}
$$

$i$ is any lattice site, and $j$ runs over the whole grid. All our simulations were done on $L$ by $L$ lattices, $L$ even, with periodic boundary conditions.

In order to quantify the critical slowing down we measured the integrated autocorrelation times, defined through

$$
\tau_{\text {int }, X}=\frac{1}{2} \sum_{t=0}^{\infty} C_{X X}(t),
$$




\begin{tabular}{|c|r|}
\hline obs & \multicolumn{1}{|c|}{$\tau_{\text {int }}$} \\
\hline$E_{\mathrm{I}}$ & $9.96(16)$ \\
$\chi_{\mathrm{I}}$ & $11.16(20)$ \\
$E_{\mathrm{XY}}$ & $8.36(12)$ \\
$\chi_{\mathrm{XY}}$ & $8.12(12)$ \\
\hline
\end{tabular}

Table 1: Integrated autocorrelation times on an $8 \times 8$ lattice, heat bath algorithm, $\beta=2.2$.

using the selfconsistent window method proposed in $30 . C_{X X}$ denotes the normalized autocorrelation function of the observable $X$.

Table 11 shows the integrated correlation times of the four observables defined above, for $L=8$. Here and in the following we chose $\beta=2.2$. This value is compatible with most estimates of the critical inverse temperature in the literature. Our results for $\tau_{\text {int }}$ were obtained from $10^{6}$ lattice sweeps with a heat bath algorithm. $\chi_{I}$ has the longest autocorrelations. This remains so also on other lattice sizes and also with any other algorithm that we investigated.

In the following, we shall report on results obtained with four types of algorithms. In all cases the sweeps are done in a typewriter fashion.

\section{Metropolis (M):}

At site $i$, a rotation of the spin by an angle $\theta$ is proposed, where $\theta$ is chosen with uniform distribution from the interval $[-\epsilon,+\epsilon]$. $\epsilon$ can be used to tune the acceptance rate. A Metropolis hit is accepted with probability $\min [1, \exp (-\beta \Delta E)]$, where $\Delta E$ denotes the change of energy associated with the proposed rotation. At site $i$, one performs $n_{\text {hit }}$ Metropolis hits.

\section{Metropolis Reflection (MR):}

This is a Metropolis algorithm based on the operation which lies at the heart of the Wolff cluster algorithm for the XY model. A single random unit vector $\vec{u}$ is selected. One sweeps through the lattice. At site $i$, the spin $s_{i}$ is decomposed in components parallel and perpendicular to $\vec{u}$. It is proposed to change the sign of the parallel component. The reflection is actually performed with a probability determined by the Metropolis rule.

\section{Heat Bath (HB):}

We employ a heat bath routine for the generation of $\mathrm{U}(1)$ random numbers 
developed by Hattori and Nakajima [31, based on a rejection method with transformation of variables. It has a high acceptance rate for all values of the temperature.

\section{Microcanonical Overrelaxation (OR):}

Single spins are moved such that the energy remains unchanged. The contribution to the total energy of the system that depends on a spin $\overrightarrow{s_{i}}$ is proportional to $\overrightarrow{s_{i}} \cdot \overrightarrow{h_{i}}$, where $\overrightarrow{h_{i}}$ is a weighted sum of the nearest neighbour spins of $\overrightarrow{s_{i}}$. The spin $\overrightarrow{s_{i}}$ is decomposed into components parallel and perpendicular to $\overrightarrow{h_{i}}$. The latter component is reflected (multiplied by -1 ) with probability 1 , leaving unchanged the scalar product $\overrightarrow{s_{i}} \cdot \overrightarrow{h_{i}}$. Note that the OR algorithm is non-ergodic. It can only be used in a mixture with an ergodic algorithm.

\section{Metropolis and Heat Bath Algorithms}

We started our investigation with a study of the first three algorithms to compare their efficiency. In table 2 we quote autocorrelation times for the "slowest" observable $\chi_{\mathrm{I}}$ for the Metropolis-Algorithm with various choices of parameters, for the Metropolis reflection and for the heat bath algorithm. The simulations were done at $\beta=2.2$, on an 8 by 8 lattice. We always performed one million full lattice sweeps. In the Metropolis cases we also quote acceptance rates. In all cases we measured the CPU time consumed for 20,000 sweeps on a Pentium 166 MMX PC. The last column contains a number $r_{\text {eff }}$ which is obtained by multiplying the autocorrelation time by the CPU factor, and then normalizing such that $r_{\text {eff }}$ is one for the heat bath algorithm.

The heat bath algorithm has by far the smallest autocorrelation times. However, the Metropolis reflection algorithm implementation is so much faster that it effectively becomes the most efficient algorithm among the ones investigated. In the combination with the OR algorithm to be discussed in the next section we always used two subsequent Metropolis reflection algorithm sweeps as the basic unit. Let us remark that using different algorithm implementations or computers we could have reached different conclusions.

We now investigated the critical slowing down of the Metropolis reflection algorithm by running it on various lattice sizes, always at $\beta=2.2$. Table 3 shows our findings for lattice sizes up to $L=64$. The integrated autocorrela- 


\begin{tabular}{|c||c|c|c|c|r|c|}
\hline algorithm & $\epsilon$ & $n_{\text {hit }}$ & $\tau_{\text {int }, \chi_{\mathrm{I}}}$ & acc & cpu & $r_{\text {eff }}$ \\
\hline M & 1.5 & 1 & $43.00 \pm 1.40$ & $47 \%$ & 7.1 & $0.943(31)$ \\
M & 2.5 & 1 & $34.40 \pm 1.00$ & $30 \%$ & 7.3 & $0.776(23)$ \\
M & 3.0 & 1 & $35.92 \pm 1.04$ & $26 \%$ & 7.4 & $0.821(24)$ \\
M & 3.0 & 2 & $20.40 \pm 0.44$ & $51 \%$ & 14.2 & $0.895(19)$ \\
M & 3.14 & 1 & $36.00 \pm 1.08$ & $25 \%$ & 7.4 & $0.823(25)$ \\
M & 3.14 & 2 & $21.56 \pm 0.48$ & $48 \%$ & 14.5 & $0.966(22)$ \\
MR & & & $29.68 \pm 0.56$ & $25 \%$ & 4.1 & $0.376(07)$ \\
HB & & & $11.16 \pm 0.20$ & & 29.0 & 1.000 \\
\hline
\end{tabular}

Table 2: Integrated autocorrelation times of $\chi_{\mathrm{I}}, L=8$. acc denotes the acceptance rate. cpu is a rough estimate of CPU, in seconds, for 20,000 sweeps. $r_{\text {eff }}$ is obtained from multiplying the integrated autocorrelation time by the cpu factor and normalizing such that it is one for the heat bath algorithm.

\begin{tabular}{|r||c|c|c|}
\hline$L$ & $\tau_{\text {int }, E_{\mathrm{XY}}} / L^{2.3}$ & $\tau_{\text {int }, \chi_{\mathrm{I}}} / L^{2.3}$ & $\mathrm{~S} / 10^{6}$ \\
\hline 8 & $0.1051(17)$ & $0.1306(21)$ & 1.3 \\
12 & $0.0763(17)$ & $0.1082(28)$ & 1.3 \\
16 & $0.0760(17)$ & $0.1092(27)$ & 2.5 \\
20 & $0.0753(17)$ & $0.1050(27)$ & 3.8 \\
24 & $0.0813(17)$ & $0.1112(28)$ & 6.3 \\
32 & $0.0825(17)$ & $0.1074(31)$ & 9.0 \\
40 & $0.0798(17)$ & $0.1080(38)$ & 10.0 \\
48 & $0.0796(17)$ & $0.1087(43)$ & 12.0 \\
64 & $0.0871(17)$ & $0.1117(60)$ & 13.7 \\
\hline
\end{tabular}

Table 3: Integrated autocorrelation times of $E_{\mathrm{XY}}$ and $\chi_{\mathrm{I}}$ for the Metropolis reflection algorithm, rescaled by a factor $L^{2.3}$ in both cases. The correlation times are measured in units of two sweeps. $S$ denotes the statistics in the same units. 
tion times have been rescaled by a factor $L^{2.3}$. The $\tau_{\text {int }}$ become really large. For $L=64$ we observe $\tau_{\text {int }, \chi_{\mathrm{I}}}=1593 \pm 86$. Fits with the law

$$
\tau_{\text {int }}=A \cdot L^{z}
$$

yield

\begin{tabular}{cccc} 
& $A$ & $z$ & $\chi^{2} /$ dof \\
\hline$E_{\mathrm{XY}}$ & $0.064(4)$ & $2.36(2)$ & 1.4 \\
$\chi_{\mathrm{I}}$ & $0.106(8)$ & $2.31(2)$ & 0.5 \\
\hline
\end{tabular}

2 Metropolis Reflections

In both cases the data from $L=8$ were discarded from the fit. Our error bars take into account statistical errors only.

\section{Efficiency of Overrelaxation Steps}

We decided to examine a combination of two Metropolis reflection sweeps with $N$ OR sweeps. It is a general observation that $N$ should scale like the correlation length or, if the system is at criticality, with the lattice size $L$. The latter case is relevant for us.

In order to find the right mixture of reflection and OR steps, we computed $\tau_{\text {int }}$ for the XY energy and the Ising susceptibility. The runs were done on SUN sparc stations, for lattice sizes 8, 16, and 32. Our results for the two smaller lattices are quoted in tables 4 and 5 . In the particular implementation of our code, the overrelaxation sweeps needed $\approx 1 / 16$ of the run time of two MR sweeps. This CPU factor enters into the numbers $\tau_{\text {int }}^{\text {corr }}$ defined through

$$
\tau_{\text {int }}^{\text {corr }}=\tau_{\text {int }}\left(1+\frac{N}{16}\right)
$$

The behavior of $\tau_{\text {int }}^{\text {corr }}$ in both tables together with the $L=32$ results suggest that it is reasonable to mix according to $N=\frac{3}{8} \mathrm{~L}$. Of course this would have come out differently for other CPU time ratios between the MR and the OR parts of the update procedure. On a Pentium PC, we measured a factor of $1 / 6$ between the two CPU times.

In order to study the volume dependence of the $\tau_{\text {int }}$ we made $\geq 500,000$ update cycles on various lattice sizes, ranging from $L=8$ to $L=128$. A 


\begin{tabular}{|r||c|r||r|r|}
\hline \multicolumn{1}{|c||}{$\tau_{\text {int }, E_{\mathrm{XY}}}$} & $\tau_{\text {int }, E_{\mathrm{XY}}}^{\text {corr }}$ & \multicolumn{1}{|c|}{$\tau_{\text {int }, \chi_{\mathrm{I}}}$} & \multicolumn{1}{c|}{$\tau_{\text {int }, \chi_{\mathrm{I}}}^{\text {cor }}$} \\
\hline 0 & $9.54 \pm 0.26$ & $9.54 \pm 0.26$ & $11.88 \pm 0.41$ & $11.88 \pm 0.41$ \\
1 & $5.82 \pm 0.13$ & $6.18 \pm 0.14$ & $5.63 \pm 0.12$ & $5.98 \pm 0.13$ \\
2 & $5.50 \pm 0.12$ & $6.19 \pm 0.14$ & $4.84 \pm 0.09$ & $5.45 \pm 0.10$ \\
3 & $5.39 \pm 0.11$ & $6.40 \pm 0.13$ & $4.50 \pm 0.08$ & $5.34 \pm 0.10$ \\
4 & $5.39 \pm 0.11$ & $6.74 \pm 0.14$ & $4.41 \pm 0.08$ & $5.51 \pm 0.10$ \\
5 & $5.18 \pm 0.11$ & $6.80 \pm 0.14$ & $4.21 \pm 0.07$ & $5.53 \pm 0.09$ \\
6 & $4.88 \pm 0.09$ & $6.71 \pm 0.12$ & $3.94 \pm 0.08$ & $5.42 \pm 0.11$ \\
7 & $4.79 \pm 0.09$ & $6.89 \pm 0.13$ & $3.81 \pm 0.06$ & $5.48 \pm 0.09$ \\
8 & $4.76 \pm 0.09$ & $7.14 \pm 0.14$ & $3.68 \pm 0.06$ & $5.52 \pm 0.09$ \\
\hline
\end{tabular}

Table 4: $\quad$ Integrated autocorrelation times for $E_{\mathrm{XY}}$ and $\chi_{\mathrm{I}}, L=8$.

Two MR sweeps are blended with $N$ OR sweeps, $\beta=2.2$.

\begin{tabular}{|r||r|r||c|c|}
\hline \multicolumn{1}{|c||}{} & \multicolumn{1}{|c||}{$\tau_{\text {int }, E_{\mathrm{XY}}}$} & $\tau_{\text {int, } E_{\mathrm{XY}}}^{\text {Corr }}$ & $\tau_{\text {int }, \chi_{\mathrm{I}}}$ & $\tau_{\text {int }, \chi_{\mathrm{I}}}^{\text {cor }}$ \\
\hline 0 & $32.70 \pm 1.87$ & $32.70 \pm 1.87$ & $56.94 \pm 3.95$ & $56.94 \pm 3.95$ \\
1 & $13.25 \pm 0.43$ & $14.08 \pm 0.46$ & $20.29 \pm 0.82$ & $21.56 \pm 0.87$ \\
2 & $10.57 \pm 0.31$ & $11.89 \pm 0.35$ & $13.89 \pm 0.46$ & $15.63 \pm 0.52$ \\
3 & $9.60 \pm 0.26$ & $11.40 \pm 0.31$ & $10.85 \pm 0.32$ & $12.88 \pm 0.38$ \\
4 & $9.08 \pm 0.26$ & $11.35 \pm 0.33$ & $10.24 \pm 0.30$ & $12.80 \pm 0.38$ \\
5 & $8.60 \pm 0.23$ & $11.29 \pm 0.30$ & $8.96 \pm 0.24$ & $11.76 \pm 0.32$ \\
6 & $7.79 \pm 0.19$ & $10.71 \pm 0.26$ & $7.87 \pm 0.20$ & $10.82 \pm 0.28$ \\
7 & $7.72 \pm 0.23$ & $11.19 \pm 0.33$ & $7.61 \pm 0.21$ & $10.94 \pm 0.30$ \\
8 & $7.67 \pm 0.21$ & $11.51 \pm 0.32$ & $7.38 \pm 0.17$ & $11.07 \pm 0.26$ \\
9 & $7.69 \pm 0.19$ & $12.02 \pm 0.30$ & $7.20 \pm 0.17$ & $11.25 \pm 0.27$ \\
\hline
\end{tabular}

Table 5: Same as table 4 , but for $L=16$. 


\begin{tabular}{|r||c|c|c|}
\hline$L$ & $\tau_{\text {int }, E_{\mathrm{XY}}} / L^{1.13}$ & $\tau_{\text {int }, \chi_{\mathrm{I}}} / L^{1.35}$ & $\mathrm{~S} / 10^{6}$ \\
\hline 8 & $0.514(10)$ & $0.2717(48)$ & 0.5 \\
12 & $0.430(10)$ & $0.2343(56)$ & 0.5 \\
16 & $0.340(08)$ & $0.1864(47)$ & 0.5 \\
20 & $0.341(10)$ & $0.1926(58)$ & 0.5 \\
24 & $0.322(10)$ & $0.1822(60)$ & 0.5 \\
32 & $0.289(12)$ & $0.1656(62)$ & 0.5 \\
40 & $0.296(12)$ & $0.1694(76)$ & 0.5 \\
48 & $0.290(13)$ & $0.1714(86)$ & 0.5 \\
64 & $0.262(14)$ & $0.1564(98)$ & 0.5 \\
80 & $0.291(08)$ & $0.1720(57)$ & 2.5 \\
96 & $0.297(09)$ & $0.1727(62)$ & 2.5 \\
128 & $0.288(10)$ & $0.1606(69)$ & 1.5 \\
\hline
\end{tabular}

Table 6: Integrated autocorrelation times for $E_{\mathrm{XY}}$ and $\chi_{\mathrm{I}}$ of the Metropolis reflection algorithm, blended with $N=3 L / 8$ OR sweeps. The autocorrelation times have been rescaled by factors $L^{1.13}$ and $L^{1.35}$, respectively. A unit of measurement is two Metropolis reflection sweeps combined with $3 L / 8$ OR sweeps. $S$ denotes the statistics in these units. 
cycle consisted of two MR sweeps, followed by $3 L / 8$ OR sweeps. Our results for the $\tau_{\text {int }}$ are quoted in table 6 .

Fitting with the law eq. (7), discarding lattice sizes $L \leq 24$, we obtained

\begin{tabular}{cccc} 
& $A$ & $z$ & $\chi^{2} /$ dof \\
\hline$E_{\mathrm{XY}}$ & $0.28(5)$ & $1.13(4)$ & 1.0 \\
$\chi_{\mathrm{I}}$ & $0.17(3)$ & $1.34(4)$ & 0.8 \\
\hline
\end{tabular}

2 Metropolis Reflections $+3 L / 8$ OR

For a fair comparison with the Metropolis reflection algorithm one needs to use units that take into account the work spent on the OR steps. In fair work units the scaling law is

$$
\tau_{\text {int }}=A \cdot L^{z}(1+\omega 3 L / 8),
$$

with

$$
\omega=\frac{1}{2} \frac{\mathrm{CPU} \text { cost of OR sweep }}{\mathrm{CPU} \text { cost of MR sweep }} \text {. }
$$

The asymptotic behaviour will thus be governed by an exponent $z+1$, with an amplitude $3 / 8 \omega A$. Comparing the results of the OR and the pure Metropolis algorithms, we find compatible or similar exponents. The effective amplitudes, however, differ. In case of the chiral susceptibility, the net efficiency gain for large lattices is

$$
\gamma=\frac{3}{8} \omega \frac{0.17(3)}{0.106(8)}
$$

For realistic values of $\omega$ between 0.1 and 0.2 this gives a speedup of about 10. This statement is in good agreement with the actual values of autocorrelations times observed in our simulations.

It is interesting to look also at the performance of an algorithm with a fixed admixture of OR steps. We made simulations with $N=3$ kept fixed for all lattice sizes. Our results are summarized in table 7 .

Fitting with the scaling law eq. (7), we had to discard data from $L \leq 32$. The results are

\begin{tabular}{clcc} 
& \multicolumn{1}{c}{$A$} & $z$ & $\chi^{2} /$ dof \\
\hline$E_{\mathrm{XY}}$ & $0.10(1)$ & $2.33(3)$ & 2.2 \\
$\chi_{\mathrm{I}}$ & $0.022(3)$ & $2.21(3)$ & 2.5 \\
\hline
\end{tabular}

2 Metropolis Reflections +3 OR 


\begin{tabular}{|r||c|c|c|}
\hline$L$ & $\tau_{\text {int, } E_{\mathrm{XY}}} / L^{2.32}$ & $\tau_{\text {int, }, \chi_{\mathrm{I}}} / L^{2.20}$ & $\mathrm{~S} / 10^{6}$ \\
\hline 8 & $0.04169(96)$ & $0.04391(93)$ & 0.3 \\
16 & $0.01679(39)$ & $0.02867(72)$ & 0.5 \\
20 & $0.01495(24)$ & $0.02788(55)$ & 1.3 \\
24 & $0.01419(19)$ & $0.02722(46)$ & 2.5 \\
32 & $0.01066(19)$ & $0.02280(49)$ & 2.5 \\
36 & $0.00988(20)$ & $0.02235(53)$ & 2.5 \\
40 & $0.01004(23)$ & $0.02388(66)$ & 2.5 \\
48 & $0.01029(20)$ & $0.02385(56)$ & 5.0 \\
64 & $0.00945(35)$ & $0.02080(90)$ & 2.5 \\
72 & $0.01048(27)$ & $0.02362(70)$ & 8.0 \\
96 & $0.00961(42)$ & $0.02315(18)$ & 5.0 \\
\hline
\end{tabular}

Table 7: Integrated autocorrelation times for $E_{\mathrm{XY}}$ and $\chi_{\mathrm{I}}$ for the Metropolis reflection algorithm, blended with $N=3$ OR sweeps. The autocorrelation times have been rescaled by factors $L^{2.23}$ and $L^{2.20}$, respectively. A unit of measurement is two Metropolis reflection steps combined with 3 OR steps. $S$ denotes the statistics in these units.

Assuming an $\omega$ about 0.15 , the net efficiency gain over the Metropolis reflection without OR steps is of order 3 (for $\chi_{\mathrm{I}}$ ).

\section{Conclusion}

The net efficiency gain of an inclusion of OR steps in a Monte Carlo procedure for the FFXY model might vary with implementation and fine tuning details. It does not seem to improve on the dynamical critical exponents in a significant way. However, for a given lattice size, it definitely enhances the efficiency of the updating. The fact that it can be implemented so easily makes its usage mandatory. It would be interesting to combine the OR updating with multilevel techniques. E.g., one could perform simultaneous rotations of all spins in a block of size $l$ by $l$ such that the total energy remains unchanged.

\section{References}

[1] H.S.J. van der Zant et al., Physica B 152 (1988) 56. 
[2] C. Denniston and C. Tang, Phys. Rev. Lett. 75 (1995) 3930.

[3] X.S. Ling et al., Phys. Rev. Lett. 76 (1996) 2989.

[4] J.M. Kosterlitz and D.J. Thouless, J. Phys. C 6 (1973) 1181.

[5] S. Teitel and C. Jayaprakash, Phys. Rev. B 27 (1983) 598.

[6] B. Bergé, H.T. Diep, A. Ghazali, and P. Lallemand, Phys. Rev. B 34 (1986) 3177.

[7] J.M. Thijssen and H.J.F. Knops, Phys. Rev. B 37 (1988) 7738.

[8] A.L. Scheinine, Phys. Rev. B 39 (1989) 9368.

[9] D.B. Nicolaides, J. Phys. A 24 (1991) L231.

[10] Y.M.M. Knops, B. Nienhuis, H.J.F. Knops, and H.W.J. Blöte, Phys. Rev. B 50 (1994) 1061.

[11] J.-R. Lee, Phys. Rev. B 49 (1994) 3317.

[12] S. Lee and K.-C. Lee, Phys. Rev. B 49 (1994) 15184.

[13] G. Ramirez-Santíago and J.V. José, Phys. Rev. B 49 (1994) 9567.

[14] M.P. Nightingale, E. Granato, and J.M. Kosterlitz, Phys. Rev. B 52 (1995) 7402.

[15] P. Olsson, Phys. Rev. Lett. 75 (1995) 2758.

[16] P. Olsson, Phys. Rev. B 55 (1997) 3585.

[17] R.H. Swendsen and J.S. Wang, Phys. Rev. Lett. 58 (1987) 86.

[18] U. Wolff, Nucl. Phys. B 322 (1989) 759, U. Wolff, Phys. Rev. Lett. 62 (1989) 361.

[19] D. Kandel and E. Domany, Phys. Rev. B 43 (1991) 8539.

[20] P.D. Coddington and L. Han, Phys. Rev. B 50 (1995) 3058.

[21] D. Kandel, R. Ben-Av, and E. Domany, Phys. Rev. B 45 (1992) 4700. 
[22] S.L. Adler, Phys. Rev. D 23 (1981) 2901.

[23] M. Creutz, Phys. Rev. D 36 (1987) 515.

[24] F. Brown and T.J. Woch, Phys. Rev. Lett 58 (1987) 2394.

[25] J. Apostolakis, C.F. Baillie, and G.C. Fox, Phys. Rev. D 43 (1991) 2687.

[26] C.F. Baillie and R. Gupta, Phys. Rev. B 45 (1992) 2883.

[27] U. Wolff, Phys. Lett. B 284 (1992) 94.

[28] U. Wolff, Phys. Lett. B 288 (1992) 166.

[29] I. Horváth and A.D. Kennedy, Nucl. Phys. B 510 (1998) 367.

[30] A.D. Sokal, Monte Carlo Methods in Statistical Mechanics: Foundations and New Algorithms, Cours de Troisième Cycle de la Physique en Suisse Romande, Lausanne, 1989.

[31] T. Hattori and H. Nakajima, Nucl. Phys. Proc. Suppl. 26 (1992) 635. 